\title{
A Review of Non-Alcoholic Fatty Liver Disease: From Obesity to Liver Transplant
}

Adjoa Anyane-Yeboa MD, Charmaine A. Stewart MD, FACP, AGAF

\begin{abstract}
About the Authors
Adjoa Anyane-Yeboa is the Chief Medical Resident in the Department of Internal Medicine at the University of Illinois at Chicago. Charmaine A. Stewart is an Associate Professor in the Division of Gastroenterology and Hepatology at the University of Illinois at Chicago. Correspondence may be directed to aanyane@uic.edu.
\end{abstract}

\section{Summary}

Non-alcoholic fatty liver disease (NAFLD) is becoming an increasingly common etiology of liver disease in the United States. As the prevalence of diabetes and obesity continues to increase, it will soon surpass hepatitis $\mathrm{C}$ as the most common etiology of end stage liver disease in the western world. NAFLD like many liver diseases can progress to cirrhosis, and patients often suffer morbidity secondary to the complications of portal hypertension. The exact percentage of hepatocellular carcinoma (HCC) that occurs in NAFLD patients is unknown, however, unlike many other liver diseases there is small potential for development of HCC independent of cirrhosis. Various conservative and pharmacologic therapies have been studied with varying degrees of efficacy including lifestyle modifications, bile acids, insulin sensitizers, vitamin E, and bariatric surgery. However, the only curative treatment for NAFLD cirrhosis is liver transplant. As the epidemic that is NAFLD continues to grow, more studies will need to be done to develop new therapies and curative treatments.

\section{Résumé}

La stéatose hépatique non alcoolique est une hépatopathie de plus en plus courante aux ÉtatsUnis. Au vu de la hausse continue de la prévalence du diabète et de l'obésité, elle surpassera bientôt l'hépatite $\mathrm{C}$ au rang de principale cause d'hépatopathie terminale dans la population occidentale. La stéatose hépatique non alcoolique, à l'instar de nombreuses affections hépatiques, peut évoluer vers la cirrhose, et les patients présentent souvent des affections concomitantes secondaires aux complications de l'hypertension portale. Le pourcentage exact de carcinome hépatocellulaire apparaissant chez les personnes atteintes de stéatose hépatique non alcoolique est inconnu, mais il faut savoir que, à l'encontre d'autres maladies hépatiques, il y a un faible risque de carcinome indépendant de la cirrhose. Divers traitements conservateurs et pharmacothérapies ont été étudiés, dont la modification des habitudes de vie, les acides biliaires, les insulinosensibilisateurs, la vitamine $\mathrm{E}$ et la chirurgie bariatrique; ils sont d'efficacité variable. Le seul traitement curatif de la cirrhose secondaire à la stéatose hépatique non alcoolique demeure la greffe de foie. L'épidémie de stéatose hépatique non alcoolique prenant de l'ampleur, la recherche devra se poursuivre pour aboutir à la mise au point de nouveaux traitements, notamment des traitements curatifs. 
$\mathrm{N}$ on-alcoholic fatty liver disease (NAFLD) is becoming an increasingly prevalent etiology of liver disease in the United States. Surveys conducted by the National Health and Nutrition Examination Survey (NHANES) show the prevalence of other chronic liver diseases such as hepatitis $\mathrm{B}$ virus (HBV) and hepatitis $\mathrm{C}$ virus $(\mathrm{HCV})$ has remained stable over the years, while the prevalence of NAFLD has been steadily increasing. ${ }^{1}$ NAFLD is present in approximately $30 \%$ of adults and is reported as the etiology of asymptomatic liver enzyme elevation in approximately $45-90 \%$ of patients ${ }^{2,3}$ The increasing prevalence of NAFLD mirrors the increase in the percentage of obesity, diabetes, hypertension, and the metabolic syndrome in our population. Today NAFLD is considered the hepatic equivalent of the metabolic syndrome. ${ }^{4}$ NAFLD is rapidly becoming the most common diagnosis prior to liver transplant; thus it is important to recognize the risk factors and natural history of this disease.

NAFLD includes a wide spectrum of liver pathology ranging from steatosis without inflammation to non-alcoholic steatohepatitis (NASH), advanced fibrosis, liver failure, and cirrhosis. ${ }^{5}$ Histologically, NAFLD is difficult to distinguish from alcohol-induced liver injury; thus it is necessary to ensure that individuals diagnosed with NAFLD are consuming less than 30 grams (gm) per day for men and less than 20 gm per day for women. ${ }^{6,7}$

There is a proposed "two hit" hypothesis for the development of NAFLD. The "first hit" is insulin resistance, which leads to steatosis of the liver. The "second hit" is oxidative stress induced by development of reactive oxygen species from fatty acid oxidation, which, in combination with insulin resistance, leads to NAFLD. The exact mechanism by which oxidative stress and insulin resistance predispose to NAFLD remains uncertain. ${ }^{8,9}$ However, it has been proposed that visceral adiposity and adipokines, as well as cytokines such as IL-6 and TNF-alpha, may play a role. ${ }^{10}$ Genetic susceptibility might also contribute, as shown by the increased prevalence of NAFLD in patients with the PNPLA3 polymorphism. ${ }^{11}$

\section{Risk Factors for NAFLD}

The main risk factors associated with development of NAFLD are diabetes mellitus (DM) type II, obesity, and hyperlipidemia. In a review, Miyake and colleagues noted that high body mass index, elevated alanine aminotransferase (ALT), low total bilirubin, hyperuricemia, elevated hemoglobin A1c, insulin resistance, and elevated ferritin were all associated with increased probability of developing NAFLD. ${ }^{12}$ However, others have reported the principal associated risk factors for NAFLD to be obesity and hyperlipidemia. ${ }^{6}$

Approximately $18.5 \%$ of obese individuals have been found to have histologic evidence of steatohepatitis, compared to $2.7 \%$ of lean individuals. ${ }^{13}$ Also, DM type II and obesity have been demonstrated to be associated with increasing severity of NAFLD. ${ }^{13,14}$ Other risk factors for progression to fibrosis include advanced age and inflammation on initial biopsy. ${ }^{15}$

In addition, it has been shown that patients found to have steatosis on liver biopsy tend to follow a more benign course, whereas patients with advanced fibrosis or steatohepatitis have a much poorer prognosis. The finding of steatohepatitis on histology often predicts development of cirrhosis and its liverrelated complications. ${ }^{5,15}$

\section{NAFLD and Cirrhosis}

Approximately 3-15\% of patients with NAFLD develop cirrhosis; however, this progression of NAFLD to end-stage liver disease can take decades. ${ }^{6,17,18}$ Several studies have looked at the risk factors for progression to NASH cirrhosis. In a multi-centre cross-sectional study of 1365 patients, Nakahara found poor glucose control and age to be directly associated with advanced stages of fibrosis. ${ }^{19}$ Another study cited age over 45 years, diabetes, and obesity as risk factors for progression to advanced fibrosis and cirrhosis, as well. ${ }^{5}$

Patients with NAFLD-related cirrhosis may be asymptomatic until they present with findings of advanced-stage cirrhosis, including hepatic encephalopathy, ascites, and/or variceal bleeding. ${ }^{20}$ It is often difficult to diagnose NAFLD once it has progressed to NASH cirrhosis, as steatosis frequently disappears with progression of disease. Consequently, in advanced disease, NAFLD-associated cirrhosis may be indistinguishable from other etiologies of cirrhosis. ${ }^{18}$ However, once other causes of advanced liver disease have been excluded, it is reasonable to presume that cirrhotic patients with features of the metabolic syndrome including obesity, diabetes, and hyperlipidemia have NAFLD-related cirrhosis. ${ }^{21}$

We now know that a large proportion of chronic liver disease of unknown etiology or "cryptogenic cirrhosis (cc)" is actually NAFLD-related cirrhosis. ${ }^{20,21}$ One study found that patients with cryptogenic cirrhosis have a prevalence of diabetes and obesity that is similar to patients with NASH and higher than that of patients with cirrhosis due to autoimmune or viral liver disease. ${ }^{20}$ In a study of patients who had undergone liver transplant for cryptogenic cirrhosis, NAFLD was diagnosed as the etiology of liver disease in approximately $66 \%$ of cases. ${ }^{22}$ These studies indicate the estimated percentages of NAFLDrelated cirrhosis might be underestimated, since cases of cryptogenic cirrhosis are often not taken into consideration.

Similar to other causes of cirrhosis, NAFLD-related cirrhosis is associated with hepatocellular carcinoma (HCC). Therefore, it is important to perform routine surveillance 
imaging to screen these patients for the development of HCC.

\section{NAFLD and HCC}

Traditionally, hepatocellular carcinoma (HCC) has been described predominantly in patients with cirrhosis from alcohol-induced liver injury and hepatitis B and C (with and without cirrhosis). The incidence of HCC in the US has increased approximately $80 \%$ in recent decades. Half this increase has been attributed to new cases of hepatitis $\mathrm{C}$; however, it is speculated that the remainder may be due to NAFLD. ${ }^{23}$ The exact percentage of HCC that occurs in NAFLD cirrhosis is unknown. However, the risk factors for development of HCC in NAFLD have been identified as male sex and age over 50 years. ${ }^{24}$ There is growing evidence that NAFLD, without cirrhosis, is also a risk factor for HCC. ${ }^{25}$

Multiple studies have shown that some patients with NAFLD can develop HCC in the absence of cirrhosis. Ertle et al looked at 162 patients with HCC and found that approximately $42 \%(p<0.005)$ of patients with NAFLDassociated HCC developed HCC in the absence of cirrhosis. These patients typically had features of the metabolic syndrome, including hyperlipidemia, type $2 \mathrm{DM}$, and obesity, compared to non-NAFLD patients with HCC. The authors hypothesized that the metabolic syndrome is an independent risk factor for developing HCC in NAFLD patients. ${ }^{26}$ Although NAFLD might be an independent predictor of HCC, patients with NAFLD-related cirrhosis had a higher incidence of HCC (approximately 10-13\%), compared to patients with NAFLD who did not have cirrhosis $(0.5 \%)$ after a mean follow-up of 7.5 years. ${ }^{27,28}$ Compared to other etiologies of liver disease, the overall risk of HCC in NAFLD is still lower than that of HCC from of other causes, specifically hepatitis-c related cirrhosis. ${ }^{27}$

\section{Treatment}

There are no proven therapies for NAFLD at this time; treatment is focused on management of comorbid conditions; lifestyle modifications; pharmacotherapy, including bile acids, insulin sensitizers, and vitamin E; and bariatric surgery.

\section{Lifestyle Modification}

As NAFLD is frequently associated with diabetes, obesity, and the metabolic syndrome, it should be no surprise that lifestyle modification, including weight loss, diet, and exercise remain the cornerstone of management. One study randomized obese individuals with biopsy proven NASH to an intensive lifestyle intervention group, versus a control group, and observed them over 48 weeks. The participants who lost more than $7 \%$ of their weight were shown to have significant improvements in liver histology, including improvements in steatosis, lobular inflammation, and ballooning injury. Overall, the study showed that weight reduction significantly impacts liver histology with important implications for progression of disease. ${ }^{29}$ These findings were further supported by a meta-analysis that demonstrated the effect of weight loss on liver histology in NAFLD. ${ }^{30}$ In comparison, exercise alone benefits liver steatosis but not transaminases. ${ }^{31}$ The current AASLD guidelines state that $3-5 \%$ of weight loss can improve steatosis, but up to $10 \%$ may be necessary for improvement in necroinflammation. ${ }^{32}$

\section{Pharmacotherapy}

Currently no pharmacologic therapy has been approved for use in NAFLD or NASH. Medications for weight loss, agents for hyperlipidemia, glucose-lowering agents, and antioxidants have all been investigated.

\section{Insulin Sensitizers}

Insulin sensitizers, such as metformin and the thiazolidinediones, have been proposed for treating NAFLD. Metformin is an oral biguanide used to treat DM type 2. The mechanism of action is to increase insulin sensitivity by decreasing hepatic glucose production, decreasing intestinal absorption of glucose and improving peripheral insulin uptake. ${ }^{33}$ An open-label study on metformin use in NASH patients found an initial improvement in transaminases, but only a modest improvement in hepatic steatosis and inflammation at one year (33\% and 20\%, respectively). ${ }^{34}$ Similarly, a randomized-controlled trial found no significant difference in liver histology between metformin and placebo in patients with biopsy proven NAFLD. However, the study did find that metformin led to overall improvements in weight, low-density lipoprotein (LDL) cholesterol, blood glucose levels, and hemoglobin A1c. ${ }^{35}$

Thiazolidinediones, such as pioglitazone and rosiglitazone, are peroxisome proliferator activated-receptor gamma agonists (PPAR-gamma agonists). They activate the PPAR-gamma receptor to alter the transcription of several genes involved in lipid and glucose metabolism. ${ }^{36}$ Thiazolidinediones decrease insulin resistance by increasing glucose use by tissues and decreasing glucose production. ${ }^{37} \mathrm{~A}$ meta-analysis looking at the effects of current treatments in NAFLD found that thiazolidinediones slowed progression of fibrosis and showed improvements in histologic activity and inflammation, though weight gain was a side effect in up to $75 \%$ of patients. ${ }^{38}$ Other studies found improvements in hepatic steatosis with thiazolidinediones, as well, yet they also re-demonstrated the side effect of weight gain similar to the previous study. ${ }^{15,20}$

A meta-analysis comparing thiazolidinediones and metformin in the treatment of NASH determined that the thiazolidinediones led to significant improvements in hepatic 
steatosis, hepatocyte ballooning, and alanine aminotransferase (ALT) levels, but had no significant effect on fibrosis or inflammation in patients with diabetes. However, in patients without diabetes, the thiazolidinediones showed improvement in all categories, including fibrosis. In contrast, metformin did not show any histologic or biochemical benefit. ${ }^{39}$ Metformin is currently not recommended as a treatment of liver disease in NAFLD. Pioglitazone can be used in biopsy proven NASH; however, most studies of its effectiveness were done solely in non-diabetic patients. ${ }^{32}$

\section{Bile Acids}

Bile acids have also been used to treat NAFLD. It has been hypothesized that dysregulation of bile acid transport and signalling may play a role in the pathogenesis of NAFLD. ${ }^{40}$ One study showed that ursodeoxycholic acid reduced ALT to normal levels in approximately $24.5 \%$ of patients. ${ }^{41}$ However, a systematic review from the Cochrane Review determined there is currently not enough evidence to support the use of ursodeoxycholic acid for the treatment of NAFLD; therefore, it is currently not recommended for treatment of NAFLD or NASH. ${ }^{32,42}$

\section{Vitamin E}

Vitamin $\mathrm{E}$ has also been studied in the treatment of NAFLD. Vitamin $\mathrm{E}$ is a fat-soluble antioxidant that interferes with lipid oxidation and the production of reactive oxygen species. ${ }^{43}$ Reactive oxygen species play a central role in the "two hit" hypothesis and the development of NASH, as discussed. The PIVENS trial found that vitamin $\mathrm{E}$ was associated with a significant improvement in NASH when compared to placebo. Treatment with vitamin $\mathrm{E}$ resulted in improvements in aspartate aminotransferase (AST) and ALT levels, hepatic steatosis, and lobular inflammation without change in fibrosis. ${ }^{44}$ At this time, vitamin $\mathrm{E}$ has only been found to be effective in patients who do not have diabetes. ${ }^{44,45}$ The American Association for the Study of Liver Diseases (AASLD) guidelines state that vitamin E improves histology in non-diabetic patients with NASH and can be used at a dose of $800 \mathrm{IU} /$ day in this population. ${ }^{32}$ However, the use of vitamin E remains controversial, as several studies have shown an increased risk of all-cause mortality when vitamin $\mathrm{E}$ is used in doses greater than $400 \mathrm{IU} /$ day. Thus it should be used with caution. ${ }^{46,47}$

\section{Bariatric Surgery}

The effects of bariatric surgery in the treatment of NAFLD have also been assessed. Klein et al found that patients who lost approximately $29 \%$ of their initial body weight after gastric bypass surgery had a decrease in adipose tissue lipolysis, endogenous glucose production, secretion of very-low density lipoprotein and steatosis, as well as a decrease in the mediators associated with the development and progression of fibrosis. ${ }^{48}$ Another study evaluated 18 patients two years after undergoing Roux-en-y gastric bypass and discovered resolution of steatosis in $84 \%$ and resolution of fibrosis in $75 \%$ of those who lost over 60 percent of their weight. ${ }^{49}$ Weight loss after gastric bypass surgery has been associated with improved blood glucose control, steatosis, lobular inflammation, and fibrosis, as well as resolution of liver disease in approximately $89 \% .^{50}$ Nevertheless, for patients with NAFLD who have progressed to cirrhosis with decompensation, liver transplant is the treatment of choice.

\section{Liver Transplant in NASH}

As the number of patients with NAFLD who progress to cirrhosis rises, liver transplant is becoming an increasingly important intervention. ${ }^{51}$ End-stage liver disease from cryptogenic cirrhosis accounts for approximately $7-14 \%$ of liver transplants. ${ }^{52}$

The assessment of patients with NAFLD for liver transplant involves most of the same workup as in other liver diseases. However, some pre-transplantation considerations are specific to NAFLD patients. For instance, as previously described, most NAFLD patients have diabetes and obesity. Diabetes mellitus has been found to be an independent predictor of poor survival after transplant. ${ }^{53}$ Multiple studies also found patients with pre-transplant diabetes to have higher morbidity and mortality than their non-diabetic counterparts, likely secondary to higher frequency of cardiovascular complications, renal failure, and infections. ${ }^{54}$ One case-control study found an increased incidence of complications involving cardiovascular, ophthalmologic, renal, musculoskeletal, hematologic, respiratory, and neurologic systems in diabetic patients with NAFLD who underwent liver transplant. They also found a higher rate of minor and major infections and malignancies post-transplant in diabetic patients. ${ }^{55}$ Given the risk of significant morbidity and mortality in diabetics, these patients should undergo thorough pretransplant cardiovascular evaluation and close evaluation of other comorbid conditions.

The amount of steatosis in the donor liver must also be considered prior to transplantation of a patient with NAFLD. Donor livers with severe steatosis (approximately $>60 \%$ ) have a larger risk of primary non-function in the allograft. Marsman et al performed a prospective study evaluating the outcomes of patients transplanted with livers with up to $30 \%$ steatosis. They found a significant decrease in four-month graft survival and two-year patient survival in patients transplanted with the steatotic livers. Limited graft survival in these patients was attributed to primary graft dysfunction or non-function. In the study, fatty infiltration was an independent predictor of poor 
outcome after transplantation. ${ }^{56}$ In addition, steatosis of the donor liver has been shown to be associated with development of de novo NAFLD after liver transplant. ${ }^{57}$ Despite these findings, due to the shortage of donor organs, it is often necessary to allow some degree of steatosis in the donor in order to use as many potential donor livers as possible..$^{58}$ Most transplant surgeons perform a biopsy of cadaveric livers prior to transplantation to determine degree of steatosis; however, this practice is controversial in living donors, as there are risks associated with the procedure. ${ }^{59}$ Currently, most transplant centres allow for approximately $10-30 \%$ steatosis in the donor liver prior to transplantation. ${ }^{60}$

It is speculated that, with the rising proportion of patients with NAFLD, liver transplants for NASH cirrhosis would surpass that of hepatitis C-cirrhosis related liver transplant. However, it appears patients with NAFLD-induced cirrhosis have lower associated MELD scores, which could therefore affect the number of patients eligible for liver transplantation. ${ }^{61}$

\section{Outcomes After Liver Transplant}

Overall, mortality after liver transplant for NAFLD patients is comparable to liver transplants due to other indications. The assessment of 54,687 liver transplant recipients from the UNOS registry found that graft survival at $1,3,5$, and 10 years for NASH patients was comparable to liver transplants for cholestatic liver disease and hepatitis $\mathrm{B}$ and better for alcoholic liver disease, hepatitis $\mathrm{C}$, and hepatocellular carcinoma. Moreover, patient survival at 1, 3, 5, and 10 years post-transplant was $89 \%, 85 \%, 84 \%$, and $84 \%$, respectively. Survival was similar to that of cholestatic disease and HBV and better than alcoholic liver disease, HCV, and HCC. ${ }^{53}$ Another study by Afzali et al evaluated 53,738 liver transplant recipients from the UNOS registry and found better survival for liver transplants due to NASH, compared to alcoholic liver disease, $\mathrm{HCV}$, and HCC and poorer survival than transplants due to cholestatic disease, HBV, and autoimmune hepatitis. ${ }^{62}$

There are varying data on recurrence of NAFLD after liver transplant; nevertheless, most studies agree that NASH patients do not frequently require re-transplantation. Patients who develop recurrence of steatosis and NAFLD typically have a higher average BMI in comparison to those who do not develop recurrence..$^{58}$ One single center study of 88 patients with NAFLD-related liver transplant found recurrence in 34 (39\%), isolated steatosis in 9 , steatohepatitis in 25 , and advanced fibrosis in 3 patients. Factors associated with recurrence included higher pre and post-transplant body mass index (BMI) and elevated post-transplant triglyceride levels. Patients with recurrence in this study were also on higher doses of steroids. ${ }^{63}$ In addition, patients with the genotype PNPLA3 rs738409-G who have undergone liver transplant have an increased likelihood of developing graft steatosis and recurrence as well. ${ }^{64}$

De novo NAFLD after liver transplant has been described in up to $31 \%$ of patients who have undergone liver transplantation. One multicentre retrospective study found a prevalence of $31.1 \%$ of NAFLD and $1.6 \%$ of NASH after liver transplantation. ${ }^{65}$ As discussed, pre-transplant steatosis is a risk factor for the development of NAFLD in post-transplant patients. Other risk factors for de novo NAFLD after transplant include use of tacrolimus, hyperlipidemia, post-transplant diabetes, and obesity. ${ }^{58}$ Unfortunately, many immunosuppressants, including corticosteroids and calcineurin inhibitors such as tacrolimus, promote development of the metabolic syndrome, which in turn can lead to the development of NAFLD

\section{Conclusion}

NAFLD is becoming an increasingly common etiology of liver disease in the US. As the prevalence of diabetes, obesity, and metabolic syndrome are increasing in our population, the frequency of NAFLD is steadily increasing as well. In certain patients, NAFLD can progress to NASH cirrhosis and place patients at risk of developing complications of end-stage liver disease. There is currently no approved medical therapy for NAFLD, and liver transplant is the only curative treatment. Therefore, more studies on NAFLD are needed in order to develop novel therapies.

\section{References}

1. Younossi ZM, Stepanova M, Afendy M, et al. Changes in the prevalence of the most common causes of chronic liver diseases in the United States from 1988 to 2008. Clin Gastroenterol Hepatol 2011;9:524-530.

2. Angulo P, Keach JC, Batts KP, et al. Independent predictors of liver fibrosis in patients with nonalcoholic steatohepatitis. Hepatology 1999;30:1356-62.

3. Daniel S, Ben-Menachem T, Vasudevan G, et al. Prospective evaluation of unexplained chronic liver transaminase abnormalities in asymptomatic and aymptomatic patients. Am J Gastroenterol 1999;10:3010-14.

4. Kim CH, Younossi ZM. Nonalcoholic fatty liver disease: a manifestation of the metabolic syndrome. Cleve Clin J Med 2008;75:721-8.

5. Angulo P. Nonalcoholic fatty liver disease. New Engl J Med 2002;346:1221-31.

6. Angulo P, Keach JC, Batts KP. Independent predictors of liver fibrosis in patients with nonalcoholic steatohepatitis. Hepatology 1999;30:1356-62.

7. Rehm J, Gmel G, Sempos CT, et al. Alcohol-related morbidity and mortality. Alcohol Res Health 2003;27:39-51.

8. Burke A, Lucey MR. Non-alcoholic fatty liver disease, non-alcoholic steatohepatitis and orthotopic liver transplantation. Am J Transplant 2004;4:686-93.

9. Day CP, James OF. Steatohepatitis: a tale of two "hits"? Gastroenterology 1998;114:842-5.

10. Musso G, Gambino R, Cassader M. Non-alcoholic fatty liver disease from pathogenesis to management: an update. Obes Rev 2010;11:430-45.

11. Romeo S, Kozlitina J, Xing C, et al. Genetic variation in PNPLA3 confers susceptibility to nonalcoholic fatty liver disease. Nat Genet 28;40:1461-5.

12. Miyake T, Kumagi T, Furukawa S, et al. Non-alcoholic fatty liver disease: factors associated with its presence and onset. J Gastroenterol Hepatol 2013(Suppl 4):71-8. 
13. Urena MA, Moreno Gonzalez E, Romero CJ, et al. An approach to the rational use of steatotic donor livers in liver transplantation." Hepatogastroenterology 1999;46:1164-73.

14. Bellentani S, Saccoccio G, Masutti F, et al. Prevalence of and risk factors for hepatic steatosis in Northern Italy. Ann Intern Med 2000;132:112-7.

15. Argo CK, Northup PG, Al-Osaimi AM, et al. Systematic review of risk factors for fibrosis progression in non-alcoholic steatohepatitis. J Hepatol 2009;51:371-9.

16. Angulo P, Lindor KD. Non-alcoholic fatty liver disease. J Gastroenterol Hepatol 2002;(17 Suppl):S186-90.

17. Evans CD, Oien KA, MacSween RN, et al. Non-alcoholic steatohepatitis: a common cause of progressive chronic liver injury? J Clin Pathol 2002;55:689-92.

18. Powell EE, Cooksley WG, Hanson R, et al. The natural history of nonalcoholic steatohepatitis: a follow-up study of forty-two patients for up to 21 years. Hepatology 1990;11:74-80.

19. Nakahara T, Hyogo H, Yoneda M, et al. Type 2 diabetes mellitus is associated with the fibrosis severity in patients with nonalcoholic fatty liver disease in a large retrospective cohort of Japanese patients. J Gastroenterol 2014;49:1477-84.

20. Caldwell SH, Oelsner DH, Iezzoni JC, et al. Cryptogenic cirrhosis: clinical characterization and risk factors for underlying disease. Hepatology 1999;29:664-9.

21. Maheshwari A, Thuluvath PJ. Cryptogenic cirrhosis and NAFLD: are they related? J Gastroenterol Hepatol 2006;101:664-8.

22. Nayak NC, Jain D, Vasdev N, et al. Etiologic types of end-stage chronic liver disease in adults: analysis of prevalence and their temporal changes from a study on native liver explants. Eur J Gastroenterol Hepatol 2012;24:1199-208.

23. Li J, Huang L, Liu CF, et al. Hepatocellular carcinoma: epidemiology, risk factors and pathogenesis. World J Gastroenterol 2008;14:4300-8.

24. Malik SM, Gupte PA, de Vera ME, et al. Liver transplantation in patients with nonalcoholic steatohepatitis-related hepatocellular carcinoma. Clin Gastroenterol Hepatol 2009;7:800-6.

25. Stickel F, Hellerbrand C. Non-alcoholic fatty liver disease as a risk factor for hepatocellular carcinoma: mechanisms and implications. Gut 2010;59:1303-7.

26. Ertle J, Dechêne A, Sowa JP, et al. Non-alcoholic fatty liver disease progresses to hepatocellular carcinoma in the absence of apparent cirrhosis. Int J Cancer 2011;128:2436-43.

27. Wanless IR, Lentz JS. Fatty liver hepatitis (steatohepatitis) and obesity: an autopsy study with analysis of risk factors. Hepatology 1990;12:1106-10.

28. White DL, Kanwal F, El-Serag HB. Association between nonalcoholic fatty liver disease and risk for hepatocellular cancer, based on systematic review. Clin Gastroenterol Hepatol 2012;10:1342-59.

29. Promrat K, Kleiner DE, Niemeier HM, et al. Randomized controlled trial testing the effects of weight loss on nonalcoholic steatohepatitis. Hepatology 2010;51:121-9.

30. Musso, G., R. Gambino, and M. Cassader. Non-alcoholic fatty liver disease from pathogenesis to management: an update. Obes Rev 2010;11:430-45.

31. Keating SE, Hackett DA, George J, et al. Exercise and non-alcoholic fatty liver disease: a systematic review and meta-analysis. J Hepatol 2012;57:157-66.

32. Chalasani N, Younossi Z, Lavine JE, et al. The diagnosis and management of non-alcoholic fatty liver disease: practice guideline by the American Gastroenterological Association, American Association for the Study of Liver Diseases, and American College of Gastroenterology. Gastroenterology 2012;107:811-26.

33. Bailey CJ. Biguanides and NIDDM. Diabetes Care 1992;15:755-72.

34. Nair S, Diehl AM, Wiseman M, et al. Metformin in the treatment of nonalcoholic steatohepatitis: a pilot open label trial. Aliment Pharmacol Ther 2004;20:23-8.

35. Haukeland JW, Konopski Z, Eggesbø HB, et al. Metformin in patients with non-alcoholic fatty liver disease: a randomized, controlled trial. Scand J Gastroenterol 2009;44:853-60.

36. Hauner H. The mode of action of thiazolidinediones. Diabetes Metab Res Rev 2002;(18 Suppl 2): S10-5.

37. Saltiel AR, Olefsky JM. Thiazolidinediones in the treatment of insulin resistance and type II diabetes. Diabetes 1996;45:1661-9.

38. Musso G, Cassader M, Rosina F, et al. Impact of current treatments on liver disease, glucose metabolism and cardiovascular risk in non-alcoholic fatty liver disease (NAFLD): a systematic review and meta-analysis of randomised trials. Diabetologia 2012;55:885-904.
39. Rakoski MO, Singal AG, Rogers MA, et al. Meta-analysis: insulin sensitizers for the treatment of non-alcoholic steatohepatitis. Aliment Pharmacol Ther 2010;32:1211-21.

40. Thuluvath PJ. When is diabetes mellitus a relative or absolute contraindication to liver transplantation Liver Transpl 2005 (11 Suppl 2):S25-9.

41. Ratziu V, de Ledinghen V, Oberti F, et al. A randomized controlled trial of high-dose ursodesoxycholic acid for nonalcoholic steatohepatitis. J Hepatol 2011;54:1011-9.

42. Orlando R, Azzalini L, Orando S, et al. Bile acids for non-alcoholic fatty liver disease and/or steatohepatitis. Cochrane Database Syst Rev 2007;1:CD005160.

43. Dufour JF. Vitamin $\mathrm{E}$ for nonalcoholic steatohepatitis: ready for prime time? Hepatology 2010;52:789-92.

44. Violi F, Cangemi R. Pioglitazone, vitamin E, or placebo for nonalcoholic steatohepatitis. New Engl J Med 2010;362:1675-85.

45. Pacana T, Sanyal AJ. Vitamin E and nonalcoholic fatty liver disease. Curr Opin Clin Nutr Metab Care 2012;15:641-8.

46. Bjelakovic G, Nikolova D, Gluud L, et al. Mortality in randomized trials of antioxidant supplements for primary and secondary prevention: systematic review and meta-analysis. JAMA 2007;297:842-57.

47. Miller ER 3rd, Pastor-Barriuso R, Dalal D, et al. Meta-analysis: high-dosage vitamin E supplementation may increase all-cause mortality. Ann Intern Med 2005;142:37-46.

48. Klein S, Mittendorfer B, Eagon JC, et al. Gastroenterology 2006;130:1564-72.

49. Furuya CK Jr, de Oliveira CP, de Mello ES, et al. Effects of bariatric surgery on nonalcoholic fatty liver disease: preliminary findings after 2 years. J Gastroenterol Hepatol 2007;22:510-4.

50. Barker KB, Palekar NA, Bowers SP, et al. Non-alcoholic steatohepatitis: effect of Roux-en-Y gastric bypass surgery. Am J Gastroenterol 2006;101:368-73.

51. Burke A, Lucey MR. Non-alcoholic fatty liver disease, non-alcoholic steatohepatitis and orthotopic liver transplantation. Am J Transplant 2004;4:686-93.

52. Ong J, Younossi ZM, Reddy V, et al. Cryptogenic cirrhosis and posttransplantation nonalcoholic fatty liver disease. Liver Transpl 2001;7:797-801.

53. Shields PL, Tang H, Neuberger JM, et al. Poor outcome in patients with diabetes mellitus undergoing liver transplantation. Transplantation 1999;68:530-5.

54. Takuma Y, Nouso K, Makino Y, et al. Outcomes after curative treatment for cryptogenic cirrhosis-associated hepatocellular carcinoma satisfying the milan criteria. J Gastroenterol Hepatol 2011;26:1417-24.

55. John PR, Thuluvath PJ. Outcome of liver transplantation in patients with diabetes mellitus: a case-control study. Hepatology 2001;34:889-95.

56. Marsman WA, Wiesner RH, Rodriguez L, et al. Use of fatty donor liver is associated with diminished early patient and graft survival. Transplantation 1996;62:1246-51.

57. Lim LG, Cheng CL, Wee A, et al. Prevalence and clinical associations of posttransplant fatty liver disease. Liver Int 2007;27:76-80.

58. Ong J, Younossi ZM. Non-alcoholic fatty liver disease after liver transplantation: a case of nurture and nature. Am J Gastroenterol 2010;105:621-3.

59. Brandhagen D, Fidler J, Rosen C. Evaluation of the donor liver for living donor liver transplantation. Liver Transpl 2003;9(10 Suppl 2):S16-28.

60. Trauner M, Claudel T, Fickert P, et al. Bile acids as regulators of hepatic lipid and glucose metabolism. Dig Dis 2010;28:220-4.

61. O'Leary JG, Landaverde C, Jennings L, et al. Patients with NASH and cryptogenic cirrhosis are less likely than those with hepatitis $\mathrm{C}$ to receive liver transplants. Clin Gastroenterol Hepato 2011;9:700-4.

62. Afzali A, Berry K, Ioannou GN. Excellent posttransplant survival for patients with nonalcoholic steatohepatitis in the United States. Liver Transpl 2012;18:29-37.

63. Dureja P, Mellinger J, Agni R. NAFLD recurrence in liver transplant recipients. Transplantation 2011;91:684-9.

64. Finkenstedt A, Auer C, Glodny B, et al. Patatin-like phospholipase domaincontaining protein 3 rs738409-G in recipients of liver transplants is a risk factor for graft steatosis. Clin Gastroenterol Hepatol 2013;11:1667-72.

65. Dumortier J, Giostra E, Belbouab S, et al. Non-alcoholic fatty liver disease in liver transplant recipients: another story of "seed and soil." Am J Gastroenterol 2010;105:613-20. 